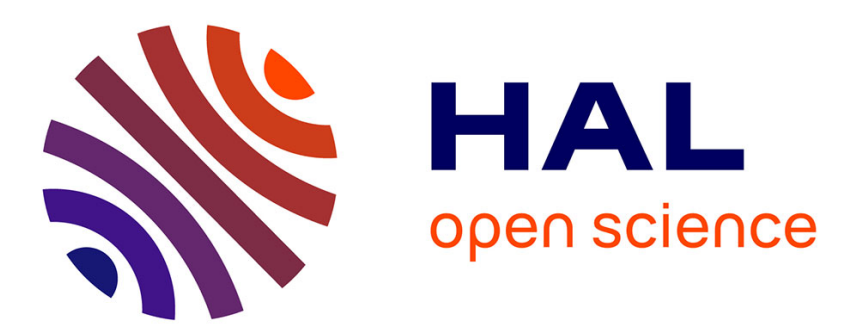

\title{
On the simultaneous stabilization of three or more plants
} Christophe Fonte, Michel Zasadzinski, Christine Bernier-Kazantsev, Mohamed Darouach

\section{To cite this version:}

Christophe Fonte, Michel Zasadzinski, Christine Bernier-Kazantsev, Mohamed Darouach. On the simultaneous stabilization of three or more plants. IEEE Transactions on Automatic Control, 2001, 46 (7), pp.1101-1107. hal-00098116

\section{HAL Id: hal-00098116 https://hal.science/hal-00098116}

Submitted on 24 Sep 2006

HAL is a multi-disciplinary open access archive for the deposit and dissemination of scientific research documents, whether they are published or not. The documents may come from teaching and research institutions in France or abroad, or from public or private research centers.
L'archive ouverte pluridisciplinaire HAL, est destinée au dépôt et à la diffusion de documents scientifiques de niveau recherche, publiés ou non, émanant des établissements d'enseignement et de recherche français ou étrangers, des laboratoires publics ou privés. 


\title{
On the Simultaneous Stabilization of Three or More Plants
}

\author{
Christophe Fonte, Michel Zasadzinski, Christine Bernier-Kazantsev \\ and Mohamed Darouach
}

\begin{abstract}
In this paper, the problem of the simultaneous stabilization of three multivariable plants is addressed. We consider the general case where the existence of a unit controller cannot be used as a sufficient condition to guarantee the existence of a simultaneous controller for three multivariable plants. The sufficient conditions given in this paper lead to a constructive controller design to stabilize simultaneously three multivariable plants. A generalization is proposed for stabilizing simultaneously $n$ multivariable plants.
\end{abstract}

\section{Keywords}

Simultaneous stabilization, Strong stabilization, Parity interlacing property, Youla parametrization.

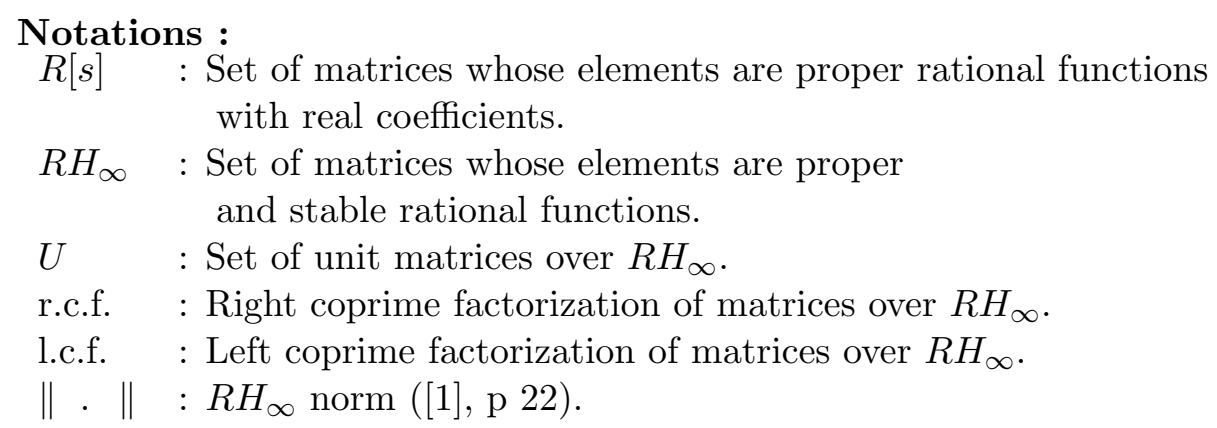

\section{INTRODUCTION}

The Youla parametrization has its origin some decades ago (see [2]) and has been successfully used to solve many theoretical problems in automatic control, like the simultaneous stabilizing controller design [3], [4], [5].

The simultaneous stabilization problem of two plants was shown in [6] to be equivalent to the stabilization of a fictitious plant by a stable controller. The stabilization of a plant by a stable controller, called strong stabilization, is fully solved by Youla et al. who provide necessary and sufficient conditions in [2]. Unlike the situation which exists for two plants, no tractable necessary and sufficient condition exists that guarantees the existence of a simultaneous stabilizing compensator for three or more linear systems. Only some sufficient conditions and some necessary conditions are given in [7], [8], [9], [10].

In [9], Wang et al. give sufficient conditions for the existence of a simultaneous compensator if, among three generalized differences between the plants to be simultaneously stabilized, two generalized differences must be a unit (see Definition 1 for the concept of generalized difference between three plants).

In [10], Wei introduces a new property, called "even interlacing property", to show that the stabilization of a plant by a stable controller having no real unstable zero is a special case of the stabilization of three plants.

C. Fonte, M. Zasadzinski and M. Darouach are with the Centre de Recherche en Automatique de Nancy (CRAN), IUT de Longwy, Université Henri-Poincaré (UHP, Nancy I), 186 rue de Lorraine, 54400 Cosneset-Romain, France. E-mail: \{fonte,mzasad,darouach\}@iut-longwy.uhp-nancy.fr.

C. Bernier-Kazantsev is with the Institut Elie Cartan, INRIA-Lorraine, Université Henri-Poincaré (UHP, Nancy I), 54506 Vandoeuvre-les-Nancy, France. E-mail: kazanc@iecn.uhp-nancy.fr. 
In [7], [8], Blondel et al. prove that the simultaneous stabilization problem of three plants is equivalent to stabilizing a fictitious plant by a unit controller if one of the three generalized differences (see Definition 1) is a unit. They point out that, in this case, the even interlacing property is not sufficient to ensure that a plant may be stabilized by a unit controller. Blondel et al. in [7], [8] also show that the even interlacing property is necessary and sufficient for the existence of a stable compensator and an inverse stable compensator that both stabilize a monovariable plant or, equivalently, that the even interlacing property is necessary and sufficient for the existence of a bistable controller providing a closed-loop with no pole on the positive real axis.

The main purpose of this paper is to study the simultaneous stabilization problem of three or more multivariable plants, without the assumption that one of the generalized differences between the plants is a unit. The paper is organized as follows. In section II we present some preliminaries on the Youla parametrization and existing results on the simultaneous stabilization of two and three plants are also reviewed. Section III presents our main results which concern the existence conditions of simultaneous compensators for three multivariable plants in the case where no generalized difference between the plants must be a unit. These existence conditions, which are sufficient, are expressed in terms of $Q$-parameters. The proposed results are illustrated by a numerical example and a design procedure is given to compute simultaneous compensators for three systems. Finally the given approach for three plants is extended to the simultaneous stabilization of $n$ plants.

\section{Preliminaries And Definitions}

\section{A. Youla parametrization and simultaneous stabilization of two plants}

Consider $(N, D)$ and $(\widetilde{D}, \widetilde{N})$ to be an r.c.f. and an l.c.f. over $R H_{\infty}$ respectively of a linear time-invariant plant $P$ (i.e. $P=N D^{-1}=\widetilde{D}^{-1} \widetilde{N}$ ). Then, for any controller $C$ that stabilizes $P$, there exist proper stable rational matrices $X, Y, \widetilde{X}$ and $\widetilde{Y}$ such that

$$
\left[\begin{array}{cc}
Y & X \\
-\widetilde{N} & \widetilde{D}
\end{array}\right]\left[\begin{array}{cc}
D & -\widetilde{X} \\
N & \widetilde{Y}
\end{array}\right]=\left[\begin{array}{ll}
I & 0 \\
0 & I
\end{array}\right]
$$

and

$$
\left[\begin{array}{cc}
D & \widetilde{X} \\
-N & \widetilde{Y}
\end{array}\right]\left[\begin{array}{cc}
Y & -X \\
\widetilde{N} & \widetilde{D}
\end{array}\right]=\left[\begin{array}{ll}
I & 0 \\
0 & I
\end{array}\right]
$$

where $(\widetilde{X}, \widetilde{Y})$ and $(Y, X)$ are an r.c.f. and an l.c.f. of a compensator $C$ which stabilizes $P$. Denote $(X N+Y D)$ by $\Phi(C, P)$ and $(\widetilde{N} \widetilde{X}+\widetilde{D} \widetilde{Y})$ by $\widetilde{\Phi}(C, P)$. The set of all stabilizing compensators of $P$ and the set of all proper plants stabilized by a given compensator $C$ can be defined as follows :

i) the set of all compensators that stabilize $P \in R[s]$ is given by $\Lambda_{1}(P)$ :

$$
\Lambda_{1}(P):=\left\{C_{g} \in R[s] / \Phi\left(C_{g}, P\right) \in U, \widetilde{\Phi}\left(C_{g}, P\right) \in U\right\},
$$

ii) the set of all plants stabilized by a given compensator $C$ is given by $\Lambda_{2}(C)$ :

$$
\Lambda_{2}(C):=\left\{P_{g} \in R[s] / \Phi\left(C, P_{g}\right) \in U, \widetilde{\Phi}\left(C, P_{g}\right) \in U\right\} .
$$

Assume that relations (1) and (2) hold, then by using a free parameter $Q \in R H_{\infty}$, we have the two following sets : the set of all stabilizing controllers for a nominal plant [1] and the set of all plants stabilizable by a given controller. 
i) For all $C_{g} \in \Lambda_{1}(P)$, there exist free parameters $Q \in R H_{\infty}, \widetilde{Q} \in R H_{\infty}$ and $V \in U$, $\widetilde{V} \in U$ such that

$$
\begin{aligned}
& \left(Y_{g}, X_{g}\right)=(V Y-Q \widetilde{N}, V X+Q \widetilde{D}) \\
& \left(\widetilde{X}_{g}, \widetilde{Y}_{g}\right)=(\widetilde{X} \widetilde{V}+D \widetilde{Q}, \widetilde{Y} \widetilde{V}-N \widetilde{Q})
\end{aligned}
$$

where $\left(Y_{g}, X_{g}\right)$ and $\left(\widetilde{X}_{g}, \widetilde{Y}_{g}\right)$ are any l.c.f. and r.c.f. of $C_{g}$ satisfying $\operatorname{det}(V Y-Q \widetilde{N}) \neq 0$ and $\operatorname{det}(\widetilde{Y} \widetilde{V}-N \widetilde{Q}) \neq 0$.

ii) For all $P_{g} \in \Lambda_{2}(C)$, there exist free parameters $Q \in R H_{\infty}, \widetilde{Q} \in R H_{\infty}$ and $V \in U$, $\widetilde{V} \in U$ such that

$$
\begin{aligned}
& \left(N_{g}, D_{g}\right)=(N V+\widetilde{Y} Q, D V-\widetilde{X} Q) \\
& \left(\widetilde{D}_{g}, \widetilde{N}_{g}\right)=(\widetilde{V} \widetilde{D}-\widetilde{Q} X, \widetilde{V} \widetilde{N}+\widetilde{Q} Y)
\end{aligned}
$$

where $\left(N_{g}, D_{g}\right)$ and $\left(\widetilde{D}_{g}, \widetilde{N}_{g}\right)$ are any r.c.f. and l.c.f. of $P_{g}$ satisfying $\operatorname{det}(D V-\widetilde{X} Q) \neq 0$ and $\operatorname{det}(\widetilde{V} \widetilde{D}-\widetilde{Q} X) \neq 0$.

Recall that a proper plant is strongly stabilizable if it is stabilizable with stable compensators (see [1], [2]), and a system satisfies the parity interlacing property (p.i.p.) if and only if it has an even number (counting multiplicities) of poles between each pair of blocking zeros on the extended positive real axis (see [1], [2]). One of the immediate and interesting consequences of the above result are the existence conditions of a simultaneous compensator for two plants.

Theorem 1: (Necessary and sufficient condition for the simultaneous stabilization of two plants) [1], [6], Let $P_{i} \in R[s], P_{j} \in R[s]$ be two plants. $P_{i}$ and $P_{j}$ are described by their associated r.c.f. and l.c.f. $\left(N_{i}, D_{i}\right),\left(N_{j}, D_{j}\right)$ and $\left(\widetilde{D}_{i}, \widetilde{N}_{i}\right),\left(\widetilde{D}_{j}, \widetilde{N}_{j}\right)$ respectively. Let $C_{i}$ be any compensator in $\Lambda_{1}\left(P_{i}\right) . C_{i}$ is described by an l.c.f. given by $\left(Y_{i}, X_{i}\right)$. The plants $P_{i}$ and $P_{j}$ are simultaneously stabilizable by a controller $C_{s i j}$ if and only if there exists $Q_{s i j} \in R H_{\infty}$ such that

$$
\left(\Phi\left(C_{i}, P_{j}\right)+Q_{s i j} \Delta_{i j}\right) \in U \text { with } \operatorname{det}\left(Y_{i}-Q_{s i j} \tilde{N}_{i}\right) \neq 0
$$

where $\Delta_{i j}$ is the generalized difference between the two plants $P_{i}$ and $P_{j}$ defined as

$$
\Delta_{i j}=\widetilde{D}_{i} N_{j}-\widetilde{N}_{i} D_{j}
$$

The generalized difference $\Delta_{i j}$ depends on the chosen factorization of the plants $P_{i}$ and $P_{j}$. However, $\Delta_{i j}$ verifies the following property : the unstable zeros of $\Delta_{i j}$ do not depend on the choice of the plant factorization. Notice that if the plants $P_{i}$ and $P_{j}$ are stable, then the generalized difference $\Delta_{i j}$ in (10) can be written as $\Delta_{i j}=P_{j}-P_{i}$ since $D_{j}$ and $\widetilde{D}_{i}$ can be chosen as $D_{j}=I$ and $\widetilde{D}_{i}=I$.

From the above theorem, note that there exists $Q_{s i j} \in R H_{\infty}$ given by (9) if and only if the fictitious plant of r.c.f. $\left(\Delta_{i j}, \Phi\left(C_{i}, P_{j}\right)\right)$ verifies the p.i.p. Substituting $Q_{s i j}$ into (5) with $V=I$, then the simultaneous stabilizing compensator $C_{s i j}$ has an l.c.f. $\left(Y_{s i j}, X_{s i j}\right)$ given by

$$
\left(Y_{s i j}, X_{s i j}\right)=\left(Y_{i}-Q_{s i j} \widetilde{N}_{i}, X_{i}+Q_{s i j} \widetilde{D}_{i}\right)
$$

An immediate consequence of the p.i.p. is that if a monovariable strongly stabilizable plant with strongly stabilizable inverse is given then this plant satisfies the even interlacing property, denoted e.i.p. The e.i.p. is defined as follows [3], [10] : a monovariable system satisfies the even interlacing property if and only if it has an even number (counting multiplicities) of poles between each pair of zeros and an even number of zeros (counting multiplicities) between each pair of poles on the extended positive real axis. 


\section{B. Some results from the literature on the simultaneous stabilization of three plants}

In the litterature some papers treat the problem of the simultaneous stabilization of $n$ SISO plants with particular constraints, (for example, see [11] for the stabilization of $n$ plants with a stable compensator). In this part, our recalls are limited to three plants and general results are given in this case.

\section{B.1 General case}

Before tackling the simultaneous stabilization problem of three plants, let us define the generalized differences between three systems.

Definition 1 (Generalized differences between three systems) Let $P_{i} \in R[s], P_{j} \in R[s]$ and $P_{k} \in R[s]$ be three plants. The plants $P_{i}, P_{j}$ and $P_{k}$ are described by their associated r.c.f. $\left(N_{i}, D_{i}\right),\left(N_{j}, D_{j}\right)$ and $\left(N_{k}, D_{k}\right)$ respectively, and by their l.c.f. $\left(\widetilde{D}_{i}, \widetilde{N}_{i}\right),\left(\widetilde{D}_{j}, \widetilde{N}_{j}\right)$ and $\left(\widetilde{D}_{k}, \widetilde{N}_{k}\right)$ respectively. The generalized differences between these three plants are defined by

$$
\Delta_{i j}=\widetilde{D}_{i} N_{j}-\widetilde{N}_{i} D_{j}, \Delta_{j k}=\widetilde{D}_{j} N_{k}-\widetilde{N}_{j} D_{k}, \Delta_{i k}=\widetilde{D}_{i} N_{k}-\widetilde{N}_{i} D_{k}
$$

A necessary and sufficient condition for simultaneously stabilizing three plants is given by the following theorem, but this condition is intractable [8].

Theorem 2: (Necessary and sufficient condition for the simultaneous stabilization of three plants) [8], [10], Let $P_{i} \in R[s], P_{j} \in R[s]$ and $P_{k} \in R[s]$ be three plants. Assume that $P_{i}, P_{j}$ and $P_{k}$ have no common intersection in the extended right half plane. The plants $P_{i}, P_{j}$ and $P_{k}$ are simultaneously stabilizable if and only if there exist three unit matrices $U_{i j}, U_{j k}$ and $U_{i k}$ such that

$$
\Delta_{i j} U_{i j}+\Delta_{j k} U_{j k}+\Delta_{i k} U_{i k}=0 .
$$

Let us now describe different cases according to whether $U_{i j}, U_{j k}$ and $U_{i k}$ are units or not and begin by considering a special case under which the determination of a simultaneous controller for three plants is easily tractable.

B.2 Assume that $\Delta_{j k} \notin U, \Delta_{i k} \notin U$ and $\Delta_{i j} \in U$

In [7], [8], the authors consider the restrictive case where $\Delta_{i j} \in U$. They have shown that the existence of a simultaneous compensator for three monovariable plants when one generalized difference is a unit (i.e. $\Delta_{i j} \in U$, see (12)), is equivalent to showing the existence of a bistable compensator for the fictitious plant of r.c.f. $\left(\Delta_{j k}, \Delta_{i k}\right)$. This result is recalled in the following theorem.

Theorem 3: (Relation between simultaneous stabilization of three plants and unit controllers) [7], [8], Assume that $\Delta_{i j} \in U$, then the monovariable plants $P_{i}, P_{j}$ and $P_{k}$ are simultaneously stabilizable if and only if the fictitious plant of r.c.f. $\left(\Delta_{j k}, \Delta_{i k}\right)$ is stabilizable by a unit controller.

In [7], [8], [10], it is stated that the examination of the e.i.p. is not sufficient for the existence of a unit controller as shown in the following theorem.

Lemma 1 (Even interlacing property) [8], [10] A monovariable plant $P$ is stabilizable by a compensator that has no zero on the positive real axis and that has stable poles if and only if $P$ verifies the e.i.p.

Indeed, as pointed out by Blondel et al. [7], a plant that satisfies the e.i.p. is stabilizable by a stable controller and by an inverse stable controller but may not be stabilizable by a unit controller.

In [7], [8] it is shown that there exists a unit controller that " $R_{+}$-stabilizes $P$ " if and only if $P$ verifies the e.i.p., where the notion of " $R_{+}$-stabilizability" is defined as follows : a monovariable plant $P \in R[s]$ is " $R_{+}$-stabilizable" if there exists a compensator $C$ such that the four transfer functions $P C(1+P C)^{-1}, P(1+P C)^{-1}, C(1+P C)^{-1},(1+P C)^{-1}$ 
have no poles on the extended positive real axis. Therefore the e.i.p. guarantees the existence of a unit controller which gives no closed-loop pole on the extended positive real axis but this property does not guarantee the existence of a unit controller that stabilizes a plant with no unstable closed-loop mode in the complex plane.

\section{MAIn RESUlts}

A. Simultaneous stabilization of three plants when $\Delta_{j k}, \Delta_{i k}$ and $\Delta_{i j}$ are not necessary in $U$

Now consider, for multivariable plants, the general case where $\Delta_{j k}, \Delta_{i k}$ and $\Delta_{i j}$ are not necessary in $U$. To derive the existence conditions for the simultaneous stabilization of three plants, two p.i.p. are examined as in section II-B.2 and a new condition is introduced in order to constrain a Youla parameter to satisfy a relationship in $U$. Therefore a simultaneous compensator for three plants will be obtained by two euclidean divisions in $R H_{\infty}$ with the remainders belonging to $U$, and the simultaneous compensator is an affine function of the quotients of these two successive divisions.

First, sufficient conditions for the simultaneous stabilization of three plants are given.

Theorem 4 (Sufficient conditions for stabilizing three plants simultaneously) Consider three plants $P_{i}, P_{j}$ and $P_{k}$ described by the r.c.f. $\left(N_{i}, D_{i}\right),\left(N_{j}, D_{j}\right),\left(N_{k}, D_{k}\right)$ and the l.c.f. $\left(\widetilde{D}_{i}, \widetilde{N}_{i}\right),\left(\widetilde{D}_{j}, \widetilde{N}_{j}\right),\left(\widetilde{D}_{k}, \widetilde{N}_{k}\right)$ respectively. Assume that

i) The plants $P_{i}$ and $P_{j}$ are simultaneously stabilizable by a compensator $C_{s i j}$ described by the associated l.c.f. and r.c.f. given by $\left(Y_{s i j}, X_{s i j}\right),\left(\widetilde{X}_{s i j}, \widetilde{Y}_{s i j}\right)$, respectively.

Notice that condition i) ensures the existence of a parameter $\widetilde{Q}_{i} \in R H_{\infty}$ satisfying the following relations

$$
\begin{aligned}
& \widetilde{\Phi}\left(C_{s i j}, P_{j}\right)^{-1} \widetilde{D}_{j}=\widetilde{\Phi}\left(C_{s i j}, P_{i}\right)^{-1} \widetilde{D}_{i}-\widetilde{Q}_{i} X_{s i j}, \\
& \widetilde{\Phi}\left(C_{s i j}, P_{j}\right)^{-1} \widetilde{N}_{j}=\widetilde{\Phi}\left(C_{s i j}, P_{i}\right)^{-1} \widetilde{N}_{i}+\widetilde{Q}_{i} Y_{s i j} .
\end{aligned}
$$

ii) There exists $Q_{s j k} \in R H_{\infty}$ such that

$$
\begin{aligned}
& \left(\Phi\left(C_{s i j}, P_{k}\right)+Q_{s j k} \Delta_{j k}\right) \in U \text { with } \operatorname{det}\left(Y_{s i j}-Q_{s j k} \widetilde{N}_{j}\right) \neq 0 \\
& \left(I-Q_{s j k} \widetilde{\Phi}\left(C_{s i j}, P_{j}\right) \widetilde{Q}_{i}\right) \in U
\end{aligned}
$$

where the parameter $\widetilde{Q}_{i}$ satisfies (14)-(15).

Then the plants $P_{i}, P_{j}$ and $P_{k}$ are simultaneously stabilizable.

Proof: Under assumption i), the plants $P_{i}$ and $P_{j}$ are simultaneously stabilizable, i.e. there exists $Q_{s i j} \in R H_{\infty}$ such that (see Theorem 1)

$$
\left(\Phi\left(C_{i}, P_{j}\right)+Q_{s i j} \Delta_{i j}\right) \in U \text { with } \operatorname{det}\left(Y_{i}-Q_{s i j} \tilde{N}_{i}\right) \neq 0
$$

where $C_{i}$ is any compensator belonging to $\Lambda_{1}\left(P_{i}\right)$ and is defined by the l.c.f. $\left(Y_{i}, X_{i}\right)$. A simultaneous compensator $C_{s i j}$ for these two plants may be described by the l.c.f. $\left(Y_{s i j}, X_{s i j}\right)$ given by

$$
\left(Y_{s i j}, X_{s i j}\right)=\left(Y_{i}-Q_{s i j} \widetilde{N}_{i}, X_{i}+Q_{s i j} \widetilde{D}_{i}\right)
$$

where $Q_{s i j} \in R H_{\infty}$. Denote by $\left(\widetilde{X}_{s i j}, \widetilde{Y}_{s i j}\right)$ an r.c.f. of this simultaneous compensator $C_{s i j}$.

Now consider assumption ii). Then there exists $Q_{s j k} \in R H_{\infty}$ such that relation (16) holds. Relation (16) is equivalent to assuming that the fictitious plant of r.c.f. $\left(\Delta_{j k}, \Phi\left(C_{s i j}, P_{k}\right)\right)$ verifies the p.i.p. Therefore the plants $P_{j}$ and $P_{k}$ are simultaneously 
stabilizable. Since $C_{s i j}$ belongs to $\Lambda_{1}\left(P_{j}\right)$, a simultaneous compensator $C_{s j k}$ stabilizing $P_{j}$ and $P_{k}$ may be described by the following l.c.f.

$$
\left(Y_{s j k}, X_{s j k}\right)=\left(Y_{s i j}-Q_{s j k} \widetilde{N}_{j}, X_{s i j}+Q_{s j k} \widetilde{D}_{j}\right) .
$$

Since the compensator $C_{s i j}$ stabilizes the plants $P_{i}$ and $P_{j}$, i.e. $P_{i} \in \Lambda_{2}\left(C_{s i j}\right)$ and $P_{j} \in \Lambda_{2}\left(C_{s i j}\right)$, there exists $\widetilde{Q}_{i} \in R H_{\infty}$ such that relations (14) and (15) hold. Inserting (14) and (15) in relation (20) gives

$$
\begin{aligned}
Y_{s j k} & =\left(I-Q_{s j k} \widetilde{\Phi}\left(C_{s i j}, P_{j}\right) \widetilde{Q}_{i}\right) Y_{s i j}-Q_{s j k} \widetilde{\Phi}\left(C_{s i j}, P_{j}\right) \widetilde{\Phi}\left(C_{s i j}, P_{i}\right)^{-1} \widetilde{N}_{i} \\
X_{s j k} & =\left(I-Q_{s j k} \widetilde{\Phi}\left(C_{s i j}, P_{j}\right) \widetilde{Q}_{i}\right) X_{s i j}+Q_{s j k} \widetilde{\Phi}\left(C_{s i j}, P_{j}\right) \widetilde{\Phi}\left(C_{s i j}, P_{i}\right)^{-1} \widetilde{D}_{i}
\end{aligned}
$$

Using (17), the controller of l.c.f. $\left(Y_{s j k}, X_{s j k}\right)$ that stabilizes simultaneously $P_{j}$ and $P_{k}$ also stabilizes $P_{i}$.

According to assumption ii) of Theorem 4 , the problem to be solved can be stated as follows : find $Q_{s j k} \in R H_{\infty}$ such that both (16) and (17) are simultaneously satisfied. A simple condition on $Q_{s j k}$ is proposed in the following lemma in order to verify (17).

Lemma 2: If there exists $Q_{s j k} \in R H_{\infty}$ such that $\left\|Q_{s j k}\right\|<\beta^{-1}$ with $\left\|\widetilde{\Phi}\left(C_{s i j}, P_{j}\right) \widetilde{Q}_{i}\right\|=\beta$ and $\widetilde{Q}_{i} \in R H_{\infty}$, then $\left(I-Q_{s j k} \widetilde{\Phi}\left(C_{s i j}, P_{j}\right) \widetilde{Q}_{i}\right) \in U$.

Proof: Assume that $\left\|Q_{s j k}\right\|<\beta^{-1}$ and $\left\|\widetilde{\Phi}\left(C_{s i j}, P_{j}\right) \widetilde{Q}_{i}\right\|=\beta$, then the following inequality

$$
\left\|Q_{s j k}\right\|\left\|\widetilde{\Phi}\left(C_{s i j}, P_{j}\right) \widetilde{Q}_{i}\right\|<1
$$

holds. Now consider the expression $\left(I-Q_{s j k} \widetilde{\Phi}\left(C_{s i j}, P_{j}\right) \widetilde{Q}_{i}\right)$ and define $U_{1}=I$ and $U_{2}=$ $\left(I-Q_{s j k} \widetilde{\Phi}\left(C_{s i j}, P_{j}\right) \widetilde{Q}_{i}\right)$, then we have $\left\|U_{1}-U_{2}\right\|<1$. From [1] (pp 22-23), $\left\|U_{1}-U_{2}\right\|<$ $\left\|U_{1}^{-1}\right\|$ implies $\left(I-Q_{s j k} \widetilde{\Phi}\left(C_{s i j}, P_{j}\right) \widetilde{Q}_{i}\right) \in U$.

From assumption i) of Theorem 4, we have $\widetilde{\Phi}\left(C_{s i j}, P_{j}\right) \in U$ where $C_{s i j}$ is a simultaneous stabilizing compensator for $P_{i}$ and $P_{j}$ which can be determined using a method proposed in $[1],[5],[6]$.

Therefore the assumption i) of Theorem 4 and relations (16) and (23) can be easily reformulated in order to derive a simultaneous controller for three plants with the same conditions.

Lemma 3 (Simultaneous controller for three plants) Consider three plants $P_{i}, P_{j}$ and $P_{k}$ described by the r.c.f. $\left(N_{i}, D_{i}\right),\left(N_{j}, D_{j}\right),\left(N_{k}, D_{k}\right)$ and the l.c.f. $\left(\widetilde{D}_{i}, \widetilde{N}_{i}\right),\left(\widetilde{D}_{j}, \widetilde{N}_{j}\right),\left(\widetilde{D}_{k}, \widetilde{N}_{k}\right)$ respectively and consider the two following assumptions :

i) there exists a compensator $C_{s i j} \in \Lambda_{1}\left(P_{i}\right) \cap \Lambda_{1}\left(P_{j}\right)$,

ii) there exists $Q_{s j k} \in R H_{\infty}$ satisfying (16) such that $\left\|Q_{s j k}\right\|\left\|\widetilde{\Phi}\left(C_{s i j}, P_{j}\right) \widetilde{Q}_{i}\right\|<1$ where $\widetilde{Q}_{i} \in R H_{\infty}$ satisfies (14)-(15).

Then, the three plants $P_{i}, P_{j}$ and $P_{k}$ have a simultaneous stabilizing compensator $C_{s j k}$ described by the following l.c.f. $\left(Y_{s j k}, X_{s j k}\right)$

$$
\left(Y_{s j k}, X_{s j k}\right)=\left(Y_{s i j}-Q_{s j k} \widetilde{N}_{j}, X_{s i j}+Q_{s j k} \widetilde{D}_{j}\right)
$$

where $\operatorname{det}\left(Y_{s i j}-Q_{s j k} \widetilde{N}_{j}\right) \neq 0$ and $\left(Y_{s i j}, X_{s i j}\right)$ is an l.c.f. of $C_{s i j}$.

Proof: Obvious from Theorem 4 and Lemma 2.

\section{B. Illustrative example}

In order to illustrate the conditions given in Theorem 4 and Lemma 3, we consider example 2 of [11]. 
Let $P_{i}, P_{j}$ and $P_{k}$ be three proper plants

$$
P_{i}=\frac{-s+2}{\left(s^{2}-1\right)(s+2)}, \quad P_{j}=\frac{-s+2}{s^{2}(s+2)}, \quad P_{k}=\frac{-s+2}{\left(s^{2}+1\right)(s+2)}
$$

with the following r.c.f. and l.c.f.

$$
\begin{array}{rlrl}
\left(N_{i}, D_{i}\right) & =\left(\frac{-s+2}{(s+h)^{2}}, \frac{\left(s^{2}-1\right)(s+2)}{(s+h)^{2}}\right), & \left(\widetilde{D}_{i}, \widetilde{N}_{i}\right)=\left(\frac{\left(s^{2}-1\right)(s+2)}{(s+h)^{2}}, \frac{-s+2)}{(s+h)^{2}}\right), \\
\left(N_{j}, D_{j}\right)=\left(\frac{-s+2}{(s+h)^{2}}, \frac{s^{2}(s+2)}{(s+h)^{2}}\right), & \left(\widetilde{D}_{j}, \widetilde{N}_{j}\right)=\left(\frac{s^{2}(s+2)}{(s+h)^{2}}, \frac{-s+2}{(s+h)^{2}}\right), \\
\left(N_{k}, D_{k}\right)=\left(\frac{-s+2}{(s+h)^{2}}, \frac{\left(s^{2}+1\right)(s+2)}{(s+h)^{2}}\right), & \left(\widetilde{D}_{k}, \widetilde{N}_{k}\right)=\left(\frac{\left(s^{2}+1\right)(s+2)}{(s+h)^{2}}, \frac{-s+2}{(s+3)^{2}}\right)
\end{array}
$$

where $h=2.12$.

The plant $P_{i}$ is stabilized by the controller $C_{i}$ of l.c.f.

$$
\left(Y_{i}, X_{i}\right)=\left(\frac{121 s^{2}+364 s+244}{\left(s+b_{1}\right)^{2}}, \frac{s^{2}+8 s+150}{\left(s+b_{1}\right)^{2}}\right) \text { with } b_{1}=0.02 .
$$

The rational functions $\Delta_{i j}, \Delta_{j k}$ and $\Phi\left(C_{i}, P_{i}\right), \Phi\left(C_{i}, P_{j}\right)$ are given by

$$
\begin{aligned}
\Delta_{i j} & =\frac{s^{2}-4}{100 \times\left(0.01 s^{6}+0.1272 s^{5}+0.67416 s^{4}+1.9056256 s^{3}+3.029944704 s^{2}+2.5693931 s+0.9078522318\right)}, \\
\Delta_{j k} & =\frac{s^{2}-4}{100 \times\left(0.01 s^{6}+0.1272 s^{5}+0.67416 s^{4}+1.9056256 s^{3}+3.029944704 s^{2}+2.5693931 s+0.9078522318\right)},
\end{aligned}
$$

and

$$
\begin{aligned}
& \Phi\left(C_{i}, P_{i}\right)=\frac{s^{5}+10 s^{4}+44 s^{3}+168 s^{2}+318 s+188}{s^{5}+6.4 s^{4}+13.738 s^{3}+10.07 s^{2}+0.3865184 s+0.0038112512} \in U, \\
& \Phi\left(C_{i}, P_{j}\right)=\frac{s^{5}+10 s^{4}+45 s^{3}+178 s^{2}+484 s+488}{s^{5}+6.4 s^{4}+13.738 s^{3}+10.07 s^{2}+0.3865184 s+0.0038112512} \notin U .
\end{aligned}
$$

The fictitious plant of r.c.f. $\left(\Delta_{i j}, \Phi\left(C_{i}, P_{j}\right)\right)$ verifies the p.i.p., then the plants $P_{i}$ and $P_{j}$ are simultaneously stabilizable. A simultaneous compensator $C_{s i j}$ stabilizing the plants $P_{i}$ and $P_{j}$ is given by the following l.c.f. and r.c.f.

$$
\begin{aligned}
& \left(Y_{s i j}, X_{s i j}\right)=\left(\frac{121 s^{2}+364 s+244}{\left(s+b_{2}\right)^{2}}, \frac{3 s^{2}+24 s+196}{\left(s+b_{2}\right)^{2}}\right), \\
& \left(\widetilde{X}_{s i j}, \widetilde{Y}_{s i j}\right)=\left(\frac{3 s^{2}+24 s+196}{\left(s+b_{2}\right)^{2}}, \frac{121 s^{2}+364 s+244}{\left(s+b_{2}\right)^{2}}\right)
\end{aligned}
$$

where $b_{2}=0.002$. Then we obtain

$$
\begin{aligned}
\Phi\left(C_{s i j}, P_{i}\right) & =\frac{3 s^{5}+30 s^{4}+120 s^{3}+240 s^{2}+240 s+96}{s^{5}+6.364 s^{4}+13.508644 s^{3}+9.5820862 s^{2}+0.0381664448 s+0.000038112512} \in U, \\
\Phi\left(C_{s i j}, P_{j}\right) & =\frac{3 s^{5}+30 s^{4}+123 s^{3}+270 s^{2}+484 s+488}{s^{5}+6.364 s^{4}+13.508644 s^{3}+9.5820862 s^{2}+0.0381664448 s+0.000038112512} \in U, \\
\Phi\left(C_{s i j}, P_{k}\right) & =\frac{3 s^{5}+30 s^{4}+126 s^{3}+300 s^{2}+728 s+880}{s^{5}+6.364 s^{4}+13.508644 s^{3}+9.5820862 s^{2}+0.0381664448 s+0.000038112512} \notin U, \\
\widetilde{\Phi}\left(C_{s i j}, P_{j}\right) & =\Phi\left(C_{s i j}, P_{j}\right) \text { and } \widetilde{\Phi}\left(C_{s i j}, P_{i}\right)=\Phi\left(C_{s i j}, P_{i}\right) .
\end{aligned}
$$


The fictitious system of r.c.f. $\left(\Delta_{j k}, \Phi\left(C_{s i j}, P_{k}\right)\right)$ verifies the p.i.p., then the plants $P_{j}$ and $P_{k}$ are simultaneously stabilizable. A simultaneous compensator $C_{s j k}$ for the plants $P_{i}, P_{j}$ and $P_{k}$ may be given by the following l.c.f. $\left(Y_{s j k}, X_{s j k}\right)$

$$
\left(Y_{s j k}, X_{s j k}\right)=\left(\frac{121 s^{2}+364 s+244}{\left(s+b_{3}\right)^{2}}, \frac{s^{2}+8 s+196}{\left(s+b_{3}\right)^{2}}\right)
$$

with $b_{3}=2$.

Now, check the conditions given in Theorem 4 and Lemma 3. From relations (14) and (15), the parameter $\widetilde{Q}_{i}=\frac{n u m \widetilde{Q}_{i}}{\operatorname{den} \widetilde{Q}_{i}} \in R H_{\infty}$ is described by

$$
\begin{aligned}
\text { num } \widetilde{Q}_{i}= & 10^{-6} \times\left(-0.00121 s^{14}-0.01904088 s^{13}-0.12562643864 s^{12}-0.43189118766752 s^{11}-\right. \\
& 0.70695953507366 s^{10}+0.14363944483332 s^{9}+3.11582587574160 s^{8}+6.58260695694717 s^{7}+ \\
& 6.96868317943686 s^{6}+3.88504718354848 s^{5}+0.91686645989331 s^{4}+0.00718064110044 s^{3}+ \\
& \left.0.00002138818727 s^{2}+0.00000002841531 s+0.00000000001418\right), \\
\operatorname{den} \widetilde{Q}_{i}= & 0.00000001089 s^{18}+0.00000038908080 s^{17}+0.00000656953344 s^{16}+0.00006969367924 s^{15}+ \\
& 0.00052142267802 s^{14}+0.00292939778408 s^{13}+0.01285222706199 s^{12}+0.04520044035703 s^{11}+ \\
& 0.12966297409078 s^{10}+0.30651357256270 s^{9}+0.59923797995720 s^{8}+0.96587197317616 s^{7}+ \\
& 1.27079188617297 s^{6}+1.34115061962108 s^{5}+1.10549389334584 s^{4}+0.68374711641405 s^{3}+ \\
& 0.29762694764684 s^{2}+0.08108827713292 s+0.01037757897121 .
\end{aligned}
$$

The Youla parameter $Q_{s j k}=\frac{n u m Q_{s j k}}{\operatorname{den} Q_{s j k}} \in R H_{\infty}$ in relation (24) is given by

$$
\begin{aligned}
\text { num } Q_{s j k}= & \left(0.0000242 s^{15}+0.0007195208 s^{14}+0.0093898969928 s^{13}+0.0720199905065 s^{12}+\right. \\
& 0.36425726318596 s^{11}+1.28579351965368 s^{10}+3.25798828355052 s^{9}+5.98281208585831 s^{8}+ \\
& 7.91323087555328 s^{7}+7.35888298073673 s^{6}+4.57058349123792 s^{5}+1.70440681248100 s^{4}+ \\
& \left.0.29030342414984 s^{3}+0.00114090648634 s^{2}+0.00000113416164 s\right), \\
\operatorname{den} Q_{s j k}= & 0.0000003 s^{15}+0.0000078012 s^{14}+0.0001092312012 s^{13}+0.0009964368312 s^{12}+ \\
& 0.0063040844368 s^{11}+0.028896604384 s^{10}+0.09991711080040 s^{9}+0.2686297218856 s^{8}+ \\
& 0.5687949208064 s^{7}+0.9439231593216 s^{6}+1.2009784756864 s^{5}+1.1127606050048 s^{4}+ \\
& 0.6681166608384 s^{3}+0.193174351872 s^{2}+0.00076471552 s+0.00000076206080
\end{aligned}
$$

and satisfies relation (16) with

$\left(\Phi\left(C_{s i j}, P_{k}\right)+Q_{s j k} \Delta_{j k}\right)=\frac{s^{5}+10 s^{4}+92 s^{3}+280 s^{2}+696 s+880}{s^{5}+10.36 s^{4}+42.9232 s^{3}+88.900928 s^{2}+92.045312 s+38.112512} \in U$.

With the above values of $\widetilde{Q}_{i}$ and $Q_{s j k}$, the condition (23) holds, i.e.

$$
\left\|Q_{s j k}\right\|\left\|\widetilde{\Phi}\left(C_{s i j}, P_{j}\right) \widetilde{Q}_{i}\right\|=0.858<1,
$$

and relation (17) is satisfied.

C. A design procedure for the simultaneous stabilization of three plants

A tractable solution to the problem of designing controllers that stabilize three plants (established in Lemma 3) which can be used to obtain a computational framework, is given by the following algorithm :

i) Choose $\left(Y_{i}, X_{i}\right)$ an l.c.f. of a given controller $C_{i} \in \Lambda_{1}\left(P_{i}\right)$. 
ii) Compute $\Delta_{i j}$ and check if the plant of r.c.f. $\left(\Delta_{i j}, \Phi\left(C_{i}, P_{j}\right)\right)$ verifies the p.i.p.

iii) Compute the simultaneous compensator $C_{s i j}$ described by the l.c.f. $\left(Y_{s i j}, X_{s i j}\right)$.

iv) Compute $\Delta_{j k}$ and check if the plant of r.c.f. $\left(\Delta_{j k}, \Phi\left(C_{s i j}, P_{k}\right)\right)$ verifies the p.i.p.

v) Determine $\widetilde{Q}_{i}$ satisfying (14) and (15).

vi) Find $Q_{s j k}$ satisfying relations (16) and (23).

vii) Compute the simultaneous compensator $C_{s j k}$ described by the l.c.f. $\left(Y_{s j k}, X_{s j k}\right)$ given by $(24)$.

\section{Simultaneous stabilization of $n$ plants}

In this section, a generalization of Theorem 4 is given for the simultaneous stabilization of $n$ plants.

Let $P_{1} \in R[s], \ldots, P_{n-1} \in R[s], P_{n} \in R[s]$ described by their associated r.c.f. and l.c.f. $\left(N_{1}, D_{1}\right), \ldots,\left(N_{n-1}, D_{n-1}\right),\left(N_{n}, D_{n}\right)$ and $\left(\widetilde{D}_{1}, \widetilde{N}_{1}\right), \ldots,\left(\widetilde{D}_{n-1}, \widetilde{N}_{n-1}\right),\left(\widetilde{D}_{n}, \widetilde{N}_{n}\right)$ respectively, and define $\Delta_{n}=\widetilde{D}_{n-1} N_{n}-\widetilde{N}_{n-1} D_{n}$.

Theorem 5 (Sufficient conditions for stabilizing $n$ plants simultaneously) Consider $n$ plants $P_{1}, \ldots, P_{n-1}, P_{n}$ described by their associated r.c.f. and l.c.f. $\left(N_{1}, D_{1}\right), \ldots,\left(N_{n-1}, D_{n-1}\right)$, $\left(N_{n}, D_{n}\right)$ and $\left(\widetilde{D}_{1}, \widetilde{N}_{1}\right), \ldots,\left(\widetilde{D}_{n-1}, \widetilde{N}_{n-1}\right),\left(\widetilde{D}_{n}, \widetilde{N}_{n}\right)$ respectively. Assume that

i) The $n-1$ plants $P_{1}, \ldots, P_{n-1}$ are simultaneously stabilizable by a compensator $C_{(1, \ldots, n-1)}$ of 1.c.f. $\left(Y_{(1, \ldots, n-1)}, X_{(1, \ldots, n-1)}\right)$ and r.c.f. $\left(\widetilde{X}_{(1, \ldots, n-1)}, \widetilde{Y}_{(1, \ldots, n-1)}\right)$, respectively.

Note that condition i) ensures the existence of the parameters $\widetilde{Q}_{1}^{\prime}, \ldots, \widetilde{Q}_{n-3}^{\prime}, \widetilde{Q}_{n-2}^{\prime}$ belonging to $R H_{\infty}$ and satisfying the following expressions

$$
\begin{gathered}
\left\{\begin{array}{c}
\widetilde{D}_{n-1}=\widetilde{\Phi}\left(C_{(1, \ldots, n-1)}, P_{n-1}\right)\left(\widetilde{\Phi}\left(C_{(1, \ldots, n-1)}, P_{1}\right)^{-1} \widetilde{D}_{1}-\widetilde{Q}_{1}^{\prime} X_{(1, \ldots, n-1)}\right), \\
\widetilde{N}_{n-1}=\widetilde{\Phi}\left(C_{(1, \ldots, n-1)}, P_{n-1}\right)\left(\widetilde{\Phi}\left(C_{(1, \ldots, n-1)}, P_{1}\right)^{-1} \widetilde{N}_{1}+\widetilde{Q}_{1}^{\prime} Y_{(1, \ldots, n-1)}\right), \\
\vdots
\end{array}\right. \\
\left\{\begin{array}{c}
\widetilde{D}_{n-1}=\widetilde{\Phi}\left(C_{(1, \ldots, n-1)}, P_{n-1}\right)\left(\widetilde{\Phi}\left(C_{(1, \ldots, n-1)}, P_{n-3}\right)^{-1} \widetilde{D}_{n-3}-\widetilde{Q}_{n-3}^{\prime} X_{(1, \ldots, n-1)}\right), \\
\widetilde{N}_{n-1}=\widetilde{\Phi}\left(C_{(1, \ldots, n-1)}, P_{n-1}\right)\left(\widetilde{\Phi}\left(C_{(1, \ldots, n-1)}, P_{n-3}\right)^{-1} \widetilde{N}_{n-3}+\widetilde{Q}_{n-3}^{\prime} Y_{(1, \ldots, n-1)}\right),
\end{array}\right. \\
\left\{\begin{array}{c}
\widetilde{D}_{n-1},=\widetilde{\Phi}\left(C_{(1, \ldots, n-1)}, P_{n-1}\right)\left(\widetilde{\Phi}\left(C_{(1, \ldots, n-1)}, P_{n-2}\right)^{-1} \widetilde{D}_{n-2}-\widetilde{Q}_{n-2}^{\prime} X_{(1, \ldots, n-1)}\right), \\
\widetilde{N}_{n-1}=\widetilde{\Phi}\left(C_{(1, \ldots, n-1)}, P_{n-1}\right)\left(\widetilde{\Phi}\left(C_{(1, \ldots, n-1)}, P_{n-2}\right)^{-1} \widetilde{N}_{n-2}+\widetilde{Q}_{n-2}^{\prime} Y_{(1, \ldots, n-1)}\right) .
\end{array}\right.
\end{gathered}
$$

ii) There exists $Q_{n} \in R H_{\infty}$ such that

$$
\left(\Phi\left(C_{(1, \ldots, n-1)}, P_{n}\right)+Q_{n} \Delta_{n}\right) \in U \text { with } \operatorname{det}\left(Y_{(1, \ldots, n-1)}-Q_{n} \tilde{N}_{n-1}\right) \neq 0
$$

and

$$
\begin{aligned}
\left(I-Q_{n} \widetilde{\Phi}\left(C_{(1, \ldots, n-1)}, P_{n-1}\right) Q_{1}^{\prime}\right) & \in U \\
& \vdots \\
\left(I-Q_{n} \widetilde{\Phi}\left(C_{(1, \ldots, n-1)}, P_{n-1}\right) Q_{n-3}^{\prime}\right) & \in U \\
\left(I-Q_{n} \widetilde{\Phi}\left(C_{(1, \ldots, n-1)}, P_{n-1}\right) Q_{n-2}^{\prime}\right) & \in U
\end{aligned}
$$

where the parameters $\widetilde{Q}_{1}^{\prime}, \ldots, \widetilde{Q}_{n-3}^{\prime}, \widetilde{Q}_{n-2}^{\prime}$ are defined in i).

Then the $n$ plants $P_{1}, \ldots, P_{n-1}$ and $P_{n}$ are simultaneously stabilizable.

Proof: This theorem is proved by recurrence using the proof of Theorem 4 .

i) Assume that the $n-1$ plants $P_{1}, \ldots, P_{n-1}$ are simultaneously stabilizable by a compensator $C_{(1, \ldots, n-1)}$ described by an l.c.f. $\left(Y_{(1, \ldots, n-1)}, X_{(1, \ldots, n-1)}\right)$. 
ii) Assume that there exists $Q_{n} \in R H_{\infty}$ such that relation (25) holds, then the plants $P_{n-1}$ and $P_{n}$ are simultaneously stabilizable. Since $C_{(1, \ldots, n-1)}$ belongs to $\Lambda_{1}\left(P_{n-1}\right)$, a simultaneous compensator $C_{(n-1, n)}$ stabilizing $P_{n-1}$ and $P_{n}$ may be described by the following l.c.f.

$$
\left(Y_{(n-1, n)}, X_{(n-1, n)}\right)=\left(Y_{(1, \ldots, n-1)}-Q_{n} \widetilde{N}_{n-1}, X_{(1, \ldots, n-1)}+Q_{n} \widetilde{D}_{n-1}\right) .
$$

First, use assumption i) and relation (28) to rewrite the l.c.f. $\left(Y_{(n-1, n)}, X_{(n-1, n)}\right)$ given by (29) as an l.c.f. of a controller that stabilizes not only $P_{n-1}$ and $P_{n}$, but also stabilizes $P_{n-2}$.

Since the compensator $C_{(1, \ldots, n-1)}$ stabilizes the plants $P_{n-2}$ and $P_{n-1}$, (i.e. $P_{n-2} \in$ $\Lambda_{2}\left(C_{(1, \ldots, n-1)}\right)$ and $\left.P_{n-1} \in \Lambda_{2}\left(C_{(1, \ldots, n-1)}\right)\right)$, there exists $\widetilde{Q}_{n-2}^{\prime} \in R H_{\infty}$ such that the pair $\left(\widetilde{D}_{n-1}, \widetilde{N}_{n-1}\right)$ may be written as

$$
\begin{aligned}
& \widetilde{D}_{n-1}=\widetilde{\Phi}\left(C_{(1, \ldots, n-1)}, P_{n-1}\right)\left(\widetilde{\Phi}\left(C_{(1, \ldots, n-1)}, P_{n-2}\right)^{-1} \widetilde{D}_{n-2}-\widetilde{Q}_{n-2}^{\prime} X_{(1, \ldots, n-1)}\right) \\
& \widetilde{N}_{n-1}=\widetilde{\Phi}\left(C_{(1, \ldots, n-1)}, P_{n-1}\right)\left(\widetilde{\Phi}\left(C_{(1, \ldots, n-1)}, P_{n-2}\right)^{-1} \widetilde{N}_{n-2}+\widetilde{Q}_{n-2}^{\prime} Y_{(1, \ldots, n-1)}\right)
\end{aligned}
$$

Inserting (30) and (31) in relation (29) gives

$$
\begin{aligned}
Y_{(n-1, n)} & =\left(I-Q_{n} \widetilde{\Phi}\left(C_{(1, \ldots, n-1)}, P_{n-1}\right) \widetilde{Q}_{n-2}^{\prime}\right) Y_{(1, \ldots, n-1)} \\
& -Q_{n} \widetilde{\Phi}\left(C_{(1, \ldots, n-1)}, P_{n-1}\right) \widetilde{\Phi}\left(C_{(1, \ldots, n-1)}, P_{n-2}\right)^{-1} \widetilde{N}_{n-2}, \\
X_{(n-1, n)} & =\left(I-Q_{n} \widetilde{\Phi}\left(C_{(1, \ldots, n-1)}, P_{n-1}\right) \widetilde{Q}_{n-2}^{\prime}\right) X_{(1, \ldots, n-1)} \\
& +Q_{n} \widetilde{\Phi}\left(C_{(1, \ldots, n-1)}, P_{n-1}\right) \widetilde{\Phi}\left(C_{(1, \ldots, n-1)}, P_{n-2}\right)^{-1} \widetilde{D}_{n-2} .
\end{aligned}
$$

Then using (28), the controller of l.c.f. $\left(Y_{(n-1, n)}, X_{(n-1, n)}\right)$ that stabilizes simultaneously $P_{n-1}$ and $P_{n}$ also stabilizes $P_{n-2}$.

Second, we use assumption i) and relation (27) to rewrite the l.c.f. $\left(Y_{(n-1, n)}, X_{(n-1, n)}\right)$ given by $(29)$ as an l.c.f. of a controller that stabilizes not only $P_{n-1}$ and $P_{n}$, but also stabilizes $P_{n-3}$.

Since the compensator $C_{(1, \ldots, n-1)}$ stabilizes the plants $P_{n-3}$ and $P_{n-1}$, (i.e. $P_{n-3} \in$ $\Lambda_{2}\left(C_{(1, \ldots, n-1)}\right)$ and $\left.P_{n-1} \in \Lambda_{2}\left(C_{(1, \ldots, n-1)}\right)\right)$, there exists $\widetilde{Q}_{n-3}^{\prime} \in R H_{\infty}$ such that the pair $\left(\widetilde{D}_{n-1}, \widetilde{N}_{n-1}\right)$ may be written as

$$
\begin{aligned}
& \widetilde{D}_{n-1}=\widetilde{\Phi}\left(C_{(1, \ldots, n-1)}, P_{n-1}\right)\left(\widetilde{\Phi}\left(C_{(1, \ldots, n-1)}, P_{n-3}\right)^{-1} \widetilde{D}_{n-3}-\widetilde{Q}_{n-3}^{\prime} X_{(1, \ldots, n-1)}\right) \\
& \widetilde{N}_{n-1}=\widetilde{\Phi}\left(C_{(1, \ldots, n-1)}, P_{n-1}\right)\left(\widetilde{\Phi}\left(C_{(1, \ldots, n-1)}, P_{n-3}\right)^{-1} \widetilde{N}_{n-3}+\widetilde{Q}_{n-3}^{\prime} Y_{(1, \ldots, n-1)}\right)
\end{aligned}
$$

Inserting (34) and (35) in relation (29) gives

$$
\begin{aligned}
Y_{(n-1, n)} & =\left(I-Q_{n} \widetilde{\Phi}\left(C_{(1, \ldots, n-1)}, P_{n-1}\right) \widetilde{Q}_{n-3}^{\prime}\right) Y_{(1, \ldots, n-1)} \\
& -Q_{n} \widetilde{\Phi}\left(C_{(1, \ldots, n-1)}, P_{n-1}\right) \widetilde{\Phi}\left(C_{(1, \ldots, n-1)}, P_{n-3}\right)^{-1} \widetilde{N}_{n-3}, \\
X_{(n-1, n)} & =\left(I-Q_{n} \widetilde{\Phi}\left(C_{(1, \ldots, n-1)}, P_{n-1}\right) \widetilde{Q}_{n-3}^{\prime}\right) X_{(1, \ldots, n-1)} \\
& +Q_{n} \widetilde{\Phi}\left(C_{(1, \ldots, n-1)}, P_{n-1}\right) \widetilde{\Phi}\left(C_{(1, \ldots, n-1)}, P_{n-3}\right)^{-1} \widetilde{D}_{n-3} .
\end{aligned}
$$

Then using (27), the controller of l.c.f. $\left(Y_{(n-1, n)}, X_{(n-1, n)}\right)$ that stabilizes simultaneously $P_{n-1}$ and $P_{n}$ also stabilizes $P_{n-3}$. 
The same reasoning is applied to the plants $P_{n-4}, \ldots, P_{1}$. If the following conditions

$$
\begin{aligned}
\left(I-Q_{n} \widetilde{\Phi}\left(C_{(1, \ldots, n-1)}, P_{n-1}\right) \widetilde{Q}_{1}^{\prime}\right) & \in U \\
\left(I-Q_{n} \widetilde{\Phi}\left(C_{(1, \ldots, n-1)}, P_{n-1}\right) \widetilde{Q}_{n-4}^{\prime}\right) & \in U
\end{aligned}
$$

hold where $Q_{1}^{\prime}, \ldots, Q_{n-4}^{\prime}$ belong to $R H_{\infty}$, then the controller of l.c.f. $\left(Y_{(n-1, n)}, X_{(n-1, n)}\right)$ that stabilizes simultaneously $P_{n-3}, P_{n-2}, P_{n-1}$ and $P_{n}$ also stabilizes $P_{1}, \ldots, P_{n-4}$.

Consequently the controller of l.c.f. $\left(Y_{(n-1, n)}, X_{(n-1, n)}\right)$ stabilizes simultaneously $P_{1}$, $\ldots, P_{n-1}$ and $P_{n}$.

\section{Conclusion}

In this paper the problem of the simultaneous stabilization of three systems, using the factorization approach and the Youla parametrization, has been considered. Sufficient conditions have been given for the simultaneous stabilization of three plants without the constraint that one of the three generalized differences is a unit. Using these conditions, a constructive design has been derived to design a simultaneous stabilizing controller for three plants and these sufficient conditions have been extended to the simultaneous stabilization of $n$ plants.

\section{REFERENCES}

[1] M. Vidyasagar, Control System Synthesis : A Factorization Approach. Cambridge, MA: MIT Press, 1985.

[2] D.C. Youla, J.J. Bongiorno, and C.N. Lu, "Single-loop feedback stabilization of linear multivariable dynamical plants," Automatica, vol. 10, pp. 159-173, 1974.

[3] V. Blondel, Simultaneous Stabilization of Linear Systems, vol. 191 of Lecture Notes in Control and Information Sciences. Berlin: Springer-Verlag, 1994.

[4] V. Blondel, G. Campion, and M.R. Gevers, "A sufficient condition for simultaneous stabilization," IEEE Transactions on Automatic Control, vol. 38, pp. 1264-1268, 1993.

[5] C. Fonte, C. Bernier-Kazantsev and M. Zasadzinski, "An algebraic design for the simultaneous stabilization of two systems," Research Report 3043, INRIA, France, 1996.

[6] M. Vidyasagar, and N. Viswanadham, "Algebraic design techniques for reliable stabilization," IEEE Transactions on Automatic Control, vol. 27, pp. 1085-1095, 1982.

[7] V. Blondel, M.R. Gevers, R. Mortini and R. Rupp, "Stabilizable by a stable and by an inverse stable but not by a stable and inverse," in Proc. 31st IEEE Conference on Decision and Control, Tucson, AZ, 1992.

[8] V. Blondel, M.R. Gevers, R. Mortini, and R. Rupp, "Simultaneous stabilization of three or more plants conditions on the positive real axis do not suffice," SIAM Journal of Control and Optimization, vol. 32, pp. 572-590, 1994.

[9] S. Wang, and F.W. Fairman, "On the simultaneous stabilization of three plants," International Journal of Control, vol. 59, pp. 1095-1106, 1994.

[10] K. Wei, "The solution of a transcendental problem and its application in simultaneous stabilization problems," IEEE Transactions on Automatic Control, vol. 37, pp. 1305-1315, 1992.

[11] C.T. Abdallah, P. Dorato, and M. Bredemann, "New sufficient conditions for strong simultanous stabilization," Autoatica, vol. 33, pp. 1193-1196, 1997. 